\section{Principais causas de óbitos infantis pós- neonatais em Belo Horizonte, Minas Gerais, Brasil, 1996 a 2004}

\author{
Leading causes of post-neonatal infant \\ deaths in Belo Horizonte, State of Minas \\ Gerais, Brazil, 1996 to 2004
}

Adriana Cherem Alves 1

Elisabeth França 2

Marislaine Lumena de Mendonça 3

Edna Maria Rezende 4

Lenice Harumi Ishitani 5

Maria da Conceição Juste Werneck Côrtes 6

1 Projeto de Extensão "Prevenção do Óbito Infantil e Perinatal em Belo Horizonte". Faculdade de Medicina. Universidade Federal de Minas Gerais. Av. Prof. Alfredo, Balena, 190. Belo Horizonte, MG, Brasil. CEP: 30.130-100. E-mail: dri_cherem@yahoo.com.br 2,6 Departamento de Medicina Preventiva e Social. Faculdade de Medicina. Universidade Federal de Minas Gerais. Belo Horizonte, MG, Brasil.

3,5 Secretaria Municipal de Saúde de Belo Horizonte. Comitê de Prevenção do Óbito Fetal e Infantil. Belo Horizonte, MG, Brasil. 4 Departamento de Enfermagem Materno-Infantil e Saúde Pública. Escola de Enfermagem. Universidade Federal de Minas Gerais. Belo Horizonte, MG, Brasil.

\section{Resumo}

Objetivos: analisar a evolução das principais causas

Objectives: to analyze the evolution of the leading causes of post-neonatal mortality by underlying cause of death in the city of Belo Horizonte, Minas Gerais Brazil, between 1996 and 2004.

Methods: causes of death were grouped according to the chapters of the International Statistical Classification of Diseases and Related Health Problems, 10th review (ICD-10) and an abbreviated ICD-10 list. Diarrhea, pneumonia, malnutrition and septicemia were analyzed in a specific group for avoidable causes. Rates and proportional mortality by cause were calculated for three-year periods.

Results: 2,010 post-neonatal deaths were registered in the period, and the tendency was for the number to decrease. Infant mortality rate dropped from 26.6 to 13.3/1000 live births (LB), and postneonatal from 8.6 to 4.3/1000 LB between 1996 and 2004. Mortality rates from both respiratory and infectious diseases were down 60\%. However, the diarrhea-pneumonia-malnutrition-septicemia group was still responsible for a quarter of all deaths in 20022004. Congenital malformations have become the leading post-neonatal cause of death, along with perinatal conditions, responsible for $34 \%$ of deaths. In addition, an increase in the proportion of mortality accounted for by injuries and ill-defined causes was observed.

Conclusions: a change was observed in the profile of the causes of post-neonatal mortality in Belo Horizonte, with an increase in the risk of death from congenital anomalies and perinatal conditions. This increase may be a consequence of displacement of neonatal deaths to the postneonatal component.

Key words Infant mortality, Causes of death Minas Gerais, Brasil, entre 1996 e 2004.

Métodos: as causas de morte foram agrupadas pelos capítulos e por lista condensada da Classificação Estatística Internacional de Doenças e Problemas Relacionados à Saúde, $10 \underline{a}$ revisão - (CID-10). As diarréias, pneumonias, desnutrição e septicemia foram analisadas como grupamento específico de causas evitáveis. Taxas e mortalidade proporcional por causa foram calculadas para periodos trienais.

Resultados: foram registrados 2010 óbitos pósneonatais no periodo, com tendência decrescente. A taxa de mortalidade infantil passou de 26,6 para 13,3/1000 nascidos vivos (NV), e a pós-neonatal de 8,6 para 4,3/1000 NV de 1996 a 2004, com maior redução nas taxas por doenças respiratórias e doenças infecciosas. Apesar do decréscimo, o grupamento diarréia-pneumonia-desnutrição-septicemia ainda foi responsável por cerca de um quarto dos óbitos em 2002-2004. As malformações congênitas passaram a representar a principal causa de morte pós-neonatal no último triênio e, juntamente com as afecções perinatais, representaram 34\% dos óbitos. Observou-se ainda aumento da proporção de óbitos por causas mal definidas e por causas externas.

Conclusões: foram observadas mudanças importantes do perfil de causas de mortalidade pós-neonatal em Belo Horizonte, com aumento da participação das malformações congênitas e afecções perinatais. Esse aumento provavelmente se deveu ao deslocamento de óbitos neonatais para o periodo pós-neonatal.

Palavras-chave Mortalidade infantil, Causas de morte de mortes infantis pós-neonatais em Belo Horizonte, 


\section{Introdução}

A mortalidade infantil é reconhecida como um sensível indicador da situação de saúde de uma população e é determinada principalmente pelas condições socioeconômicas. Embora a redução das taxas de mortalidade infantil (TMI) tenha sido observada nas últimas décadas em todo mundo, ainda persistem altos índices nos países sub-desenvolvidos. ${ }^{1} \mathrm{Algu}-$ mas medidas de saúde pública têm se destacado para o aumento da sobrevida infantil, como a melhoria da atenção ao pré-natal e ao parto, o acesso aos avanços tecnológicos, o uso da terapia de reidratação oral, o incentivo ao aleitamento materno, a atenção às doenças prevalentes na infância e a ampliação da cobertura vacinal.1,2

No Brasil, a TMI passou de 33,7/1000 nascidos vivos em 1996 para 22,6/1000 nascidos vivos em 2004.3 Apesar do declínio registrado, a TMI é ainda elevada, e sua redução constitui um desafio para os serviços de saúde e para a sociedade, já que a maioria dessas mortes precoces podem ser evitadas. 4

As causas de mortalidade infantil no Brasil também sofreram modificações. A partir dos anos 80 , observou-se declínio das doenças infecciosas associadas à desnutrição e aumento das afecções perinatais, atualmente responsáveis por mais de $50 \%$ dos óbitos no primeiro ano de vida. 5

Em Belo Horizonte, Minas Gerais, a TMI vem diminuindo, seguindo a tendência dos estados brasileiros.5,6 Esse declínio foi mais acentuado a partir de 1994, principalmente devido à redução do componente de mortalidade pós-neonatal.6 É provável que essa redução tenha ocorrido, em parte, pelas ações desenvolvidas desde 1994 pela Secretaria de Saúde do Município, através do Projeto Vida, com reorganização da assistência à gestante e à criança, que incluía o acompanhamento dos recémnascidos com maior risco de óbito. ${ }^{7}$ Em 1999, foi criada a Comissão Perinatal e, posteriormente, em 2002, o Comitê de Prevenção do Óbito Infantil e Perinatal. Esse comitê, que tem a parceria da Universidade Federal de Minas Gerais (UFMG), avalia e divulga os índices de mortalidade infantil e investiga os óbitos neonatais e fetais com peso acima de $1500 \mathrm{~g}$ e todos os pós-neonatais (exceto os com malformação congênita grave), contribuindo não só para a melhoria dos registros de óbitos, mas também adicionando informações importantes sobre as circunstâncias, causas e evitabilidade dos óbitos. 8,9 As ações de melhoria da qualidade da assistência perinatal desencadeadas pela Comissão Perinatal e pelo Comitê, com importante redução na mortalidade neonatal precoce, têm recebido reconhecimento nacional. 10 Em relação à mortalidade pós-neonatal, entretanto, verificou-se que as taxas vêm sofrendo poucas variações nos últimos anos e que ainda ocorriam muitas mortes associadas à desnutrição e doenças infecciosas, como a diarréia e a pneumonia, que são óbitos sabidamente evitáveis, ou seja, aqueles que, considerando-se a ciência e a tecnologia existentes atualmente, não deveriam ter ocorrido, independentemente do fato de as tecnologias estarem ou não acessíveis para a maioria da população naquele momento. ${ }^{11}$ Essas mortes têm sido propostas como eventos sentinela, 12 eventos cuja ocorrência indica a necessidade de investigação, pois representam um sinal de alerta em relação a prováveis problemas no acesso oportuno a serviços qualificados de saúde. 13

O objetivo do presente estudo é analisar a evolução das causas de morte pós-neonatal em Belo Horizonte no período de 1996 a 2004, em especial do grupamento infecção-desnutrição, e identificar possíveis mudanças que possam subsidiar ações para a redução desses óbitos.

\section{Métodos}

Estudaram-se os óbitos de crianças na faixa etária de 28 a 364 dias de vida, residentes em Belo Horizonte, Minas Gerais, Brasil, no período de 1996 a 2004. Foram utilizados os bancos de dados do Sistema de Informação sobre Mortalidade (SIM) e do Sistema de Informação sobre Nascidos Vivos (SINASC) obtidos na Secretaria Municipal de Saúde de Belo Horizonte.

A taxa de mortalidade infantil foi calculada como o número de óbitos de crianças no primeiro ano de vida por mil nascidos vivos, a taxa de mortalidade neonatal como o número de óbitos de crianças de 0 a 27 dias de vida por mil nascidos vivos e a taxa de mortalidade pós-neonatal como o número de óbitos de crianças de 28 a 364 dias de vida por mil nascidos vivos, ${ }^{4}$ de residentes em Belo Horizonte e em cada período estudado.

Foram calculadas a mortalidade proporcional e as taxas de mortalidade pós-neonatal por grupos de causas. Para diminuir a instabilidade das taxas, os dados foram agrupados por triênios (1996-1998, 1999-2001, 2002-2004) e calculadas as taxas médias anuais de mortalidade de cada período. Para esse cálculo, considerou-se no numerador o total de óbitos ocorridos em cada triênio e no denominador o total de nascidos vivos. Utilizou-se o programa EpiInfo 3.3, para o processamento e análise dos dados.

As causas de morte foram analisadas segundo 
duas formas de tabulação: a) Capítulos da Classificação Estatística Internacional de Doenças e Problemas Relacionados à Saúde, 10 revisão - (CID10)14; e b) Lista de tabulação CID-BR da CID-10, versão da lista básica da CID considerada mais adequada à realidade brasileira. ${ }^{15}$

A lista de tabulação CID-BR foi adaptada para uso neste estudo em 10 grupos de causas, conforme especificado a seguir: a) Pneumonias: códigos J12 a J18; b) Diarréias: códigos A00 a A09; c) Desnutrição: códigos E40 a E46; d) Septicemias: códigos A40 e A41; e) Aspiração: códigos J69, K21, W78, W79 e W84; f) Meningites/Encefalites: códigos A17, A39, G00, G03, G04, G06; g) Paralisia cerebral: código G80; h) Neoplasias/leucemias: códigos C00 a D48; i) Bronquiolite: código J21; j) Asma e Mal asmático: códigos J45, J46.

Dentre essas causas, algumas sabidamente evitáveis e intrinsecamente relacionadas, foram consideradas em dois grupamentos: G1 e G2. O primeiro grupo, G1, englobou as diarréias, pneumonias e desnutrição.16 O grupo G2 incluiu a septicemia além das causas descritas em G1. Pretendeu-se, ao incluí-la, identificar as situações em que a septicemia provavelmente representou a causa terminal de óbitos por diarréia, pneumonia ou desnutrição. 17

\section{Resultados}

No período de 1996 a 2004 observou-se redução de $50 \%$ na TMI, que passou de 26,6 para $13,3 / 1000 \mathrm{NV}$. Tanto a taxa de mortalidade neonatal quanto a pósneonatal também caíram pela metade, de 18,0 para 9,0/1000 NV e de 8,6 para 4,3/1000 NV, como se pode observar na Figura 1.

Foram registrados 2010 óbitos pós-neonatais no período estudado, com decréscimo importante do número de óbitos em cada triênio consecutivo - 890 (1996-1998), 630 (1999-2001) e 490 (2002-2004) - e conseqüente redução das taxas de mortalidade pósneonatal - 7,2, 5,4 e 4,9/1.000 NV, respectivamente.

As taxas de mortalidade pós-neonatal por doenças respiratórias (J00-J99) diminuíram 63\% (Tabela 1), principalmente devido à redução dos óbitos por pneumonia, cujas taxas passaram de $180,4 / 100.000 \mathrm{NV}$ para 38,6/100.000 NV. As taxas de mortalidade por asma/mal asmático e bronquiolite também sofreram reduções (Tabela 2). No capítulo das doenças infecciosas (códigos A00-B99), as taxas declinaram aproximadamente $60 \%$ (Tabela 1 ). Nesse grupo, as diarréias foram as que mais se destacaram, com decréscimo de 78\% (Tabela 2).

\section{Figura 1}

Taxas de mortalidade infantil e seus componentes. Belo Horizonte, Minas Gerais, 1996 a 2004.

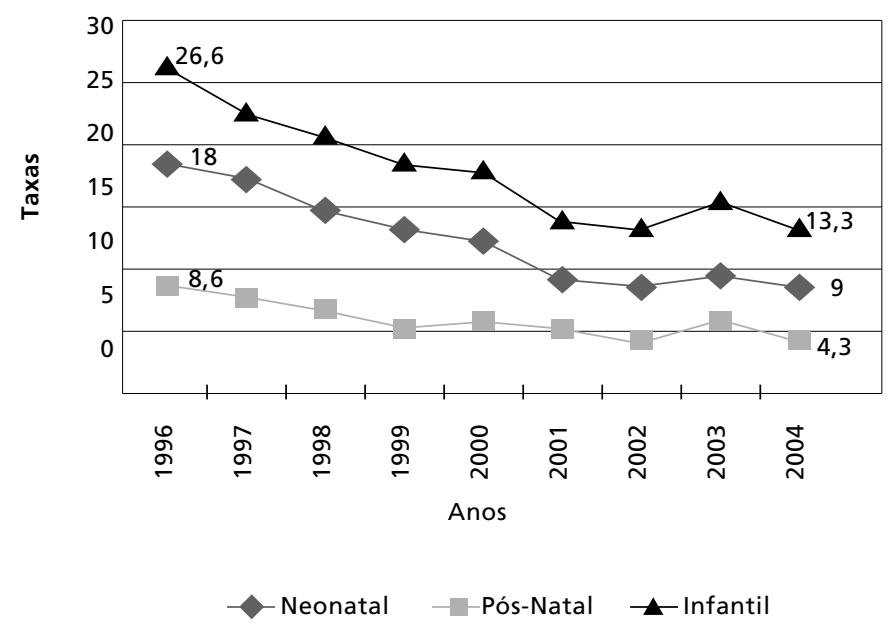

Fontes: Sistema de Informação sobre Mortalidade (SIM) e Sistema de Informação sobre Nascidos Vivos (SINASC) da Secretaria Municipal de Saúde de Belo Horizonte.

As malformações congênitas (códigos Q00-Q99) cresceram em importância, e passaram a representar a principal causa de mortalidade pós-neonatal no último triênio. Também sofreram aumento as taxas das afecções do período perinatal (CID10 P00-P93), causas mal definidas (R00-R99) e causas externas (V01-Y98) (Tabela 1). O código R99 (Outras causas mal definidas e as não especificadas de mortalidade) representou cerca de $80 \%$ do capítulo das causas mal definidas.

A taxa de mortalidade por aspiração sofreu ligeiro aumento. Em relação às meningites/encefalites e à desnutrição, observou-se uma redução de $81 \%$ e $55 \%$, respectivamente. Causas como neoplasias/leucemias e paralisia cerebral apresentaram poucos óbitos (Tabela 2).

O grupamento G1 (diarréia, pneumonia e desnutrição) teve uma redução de $76 \%$, com taxas que decresceram de 293,1 para 70,3/100.000 NV. Quanto às taxas do grupamento G2, em que se considerou a septicemia associada ao agrupamento G1, declinaram $69 \%$ - de 363,9 para $112,8 / 100.000$ NV. Entretanto, em 2002-2004, este grupo ainda representava $23 \%$ dos óbitos pósneonatais (Tabela 2). 
Mortalidade proporcional e taxas específicas por causas de mortalidade infantil pós-neonatal, segundo capítulos da 10a revisão da Classificação Internacional de Doenças (CID-10). Belo Horizonte, Minas Gerais, 1996 a 2004.

\begin{tabular}{|c|c|c|c|c|c|c|}
\hline Causas de óbito & $\begin{array}{c}1996-1998 \\
(n=890)\end{array}$ & $\begin{array}{c}1999-2001 \\
(n=630)\end{array}$ & $\begin{array}{c}2002-2004 \\
(n=490)\end{array}$ & 1996-1998 & 1999-2001 & $2002-2004$ \\
\hline Doenças do aparelho respiratório & 31,5 & 24,0 & 17,4 & 225,4 & 130,5 & 84,1 \\
\hline Malformações congênitas & 10,5 & 15,1 & 18,6 & 74,9 & 82,1 & 90,0 \\
\hline Afecções período perinatal & 9,3 & 11,1 & 15,3 & 66,8 & 60,5 & 74,2 \\
\hline Mal definidas & 3,2 & 7,5 & 9,2 & 22,5 & 40,6 & 44,5 \\
\hline Causas externas & 4,5 & 7,5 & 8,4 & 32,21 & 40,6 & 40,6 \\
\hline
\end{tabular}

Fontes: Sistema de Informação sobre Mortalidade (SIM) e Sistema de Informação sobre Nascidos Vivos (SINASC) da Secretaria Municipal de Saúde de Belo Horizonte.

Tabela 2

Mortalidade proporcional e taxas específicas por causas de mortalidade pós-neonatal, segundo lista tabular CID-BR adaptada da 10a revisão da Classificação Internacional de Doenças (CID-10). Belo Horizonte, Minas Gerais, 1996 a 2004.

\begin{tabular}{|c|c|c|c|c|c|c|}
\hline \multirow[t]{2}{*}{ Causas de óbito } & \multicolumn{3}{|c|}{ Mortalidade proporcional (\%) } & \multicolumn{3}{|c|}{ Taxas (por $100.000 \mathrm{NV}$ ) } \\
\hline & $\begin{array}{c}1996-1998 \\
(n=890)\end{array}$ & $\begin{array}{c}1999-2001 \\
(n=630)\end{array}$ & $\begin{array}{c}2002-2004 \\
(n=490)\end{array}$ & 1996-1998 & $1999-2001$ & 2002-2004 \\
\hline Pneumonias & 25,2 & 17,8 & 8,0 & 180,4 & 96,8 & 38,6 \\
\hline Sub-total (Grupo G1) & 40,9 & 26,7 & 14,5 & 293,1 & 145,2 & 70,3 \\
\hline Septicemias & 9,9 & 8,4 & 8,8 & 70,9 & 45,8 & 42,6 \\
\hline Sub-total (Grupo G2)* & 50,8 & 35,1 & 23,3 & 363,9 & 191,1 & 112,8 \\
\hline Aspiração & 4,4 & 6,0 & 6,9 & 31,3 & 32,8 & 33,6 \\
\hline Asma / Mal asmático & 1,5 & 0,9 & 1,0 & 10,4 & 5,2 & 5,0 \\
\hline Bronquiolite & 0,8 & 1,1 & 0,8 & 5,7 & 6,2 & 4,0 \\
\hline
\end{tabular}

* Grupo G2 representa a soma do Grupo G1 com as septicemias.

Fontes: Sistema de Informação sobre Mortalidade (SIM) e Sistema de Informação sobre Nascidos Vivos (SINASC) da Secretaria Municipal de Saúde de Belo Horizonte.

\section{Discussão}

Os resultados deste estudo indicam que a taxa de mortalidade infantil pós-neonatal em Belo Horizonte ainda se mantém em níveis elevados, apesar da queda de 50\% observada no período de 1996 a 2004. A taxa de 4,3/1.000 NV observada em 2004 pode ser considerada ainda muito alta, tendo em vista que nos Estados Unidos, por exemplo, essa taxa foi registrada em 1978. Em 2001, as taxas de mortalidade pós-neonatal e infantil nesse país eram de 2,3 e $6,8 / 1.000 \mathrm{NV}$, respectivamente. ${ }^{18}$

A queda da mortalidade pós-neonatal no período analisado pode ser atribuída à grande redução da mortalidade por causas respiratórias e infecciosas. Dentre as respiratórias, a taxa de mortalidade por pneumonia sofreu importante queda, provavelmente devido à melhoria do acesso aos serviços de saúde, 
manejo adequado dos casos e implantação da vacina contra Haemophilus influenzae na rede pública de saúde, importante agente causador de pneumonias nessa faixa etária. As taxas de mortalidade por asma, mal asmático e bronquiolite também sofreram importantes reduções, possivelmente em decorrência das ações do Programa de Atenção à Criança que Chia, implantado em Belo Horizonte desde 1996. Esse programa oferece vigilância e acompanhamento para as crianças asmáticas nas Unidades Básicas de Saúde. São disponibilizados medicamentos necessários para o controle da asma, inclusive medicação inalatória para as crianças portadoras de asma persistente. ${ }^{19}$

As diarréias também sofreram expressiva queda, passando de segunda para quarta causa de óbito pósneonatal, o que pode ser explicado por intervenções ambientais, como o aumento da cobertura de saneamento básico, pela utilização da terapia de reidratação oral e por ações preventivas, como o incentivo ao aleitamento materno.

A redução de 55\% nas taxas de mortalidade por desnutrição pode estar relacionada à implantação do Programa de Prevenção e Combate à Desnutrição em 1993, entre outros. 20 É provável, entretanto, que o impacto da desnutrição para a mortalidade pósneonatal possa estar subestimado, já que muitas vezes a mesma não é selecionada como causa básica do óbito, pelas regras de seleção de causa básica da CID-10 ou por não ser mencionada na Declaração de Óbito. Quando se considera o enfoque das causas múltiplas de óbito, são relatadas taxas por desnutrição cinco vezes maiores. 17

Dessa forma, neste estudo foi evidenciada uma redução importante nas taxas de mortalidade por diarréia, pneumonia, desnutrição e septicemia, os grupamentos G1 e G2. Entretanto, essas causas ainda representam uma proporção importante da mortalidade pós-neonatal, o que sugere possíveis problemas como dificuldades de acesso aos serviços de saúde qualificados.

Em contrapartida, algumas causas de morte cresceram em importância, contribuindo para a relativa estabilização das taxas de mortalidade pósneonatal nos últimos anos. As mortes por malformações congênitas aumentaram proporcionalmente ao longo do tempo e passaram a representar a principal causa de óbito no último triênio, ultrapassando as doenças respiratórias e as infecciosas. Esse fato poderia ser atribuído somente à redução das outras causas de morte. Entretanto, houve também aumento das taxas, estando provavelmente relacionado à maior sobrevida das crianças com malformações e ao deslocamento desses óbitos do período neonatal para o pós-neonatal devido à melhoria da assistência ao recém-nascido e ao maior acesso a unidades de tratamento intensivo. Apesar das mortes por malformações congênitas serem de difícil prevenção, sabese que um pré-natal de boa qualidade pode contribuir ao desaconselhar o uso de álcool e drogas e diagnosticar e tratar afecções maternas de forma adequada. Mas é difícil quantificar seu possível impacto na redução desses óbitos. ${ }^{2}$ A mortalidade proporcional por malformações congênitas em Belo Horizonte no último triênio foi muito semelhante a dos Estados Unidos em 1994 entre brancos. ${ }^{21}$ Entretanto, as taxas norte-americanas eram inferiores tanto em brancos quanto em negros e, ao contrário do observado em Belo Horizonte, estão apresentando decréscimo ao longo do tempo. 21

As mortes por afecções perinatais também aumentaram no período. É provável que isto tenha ocorrido por deslocamento de óbitos do período neonatal para o pós-neonatal. Em Belo Horizonte, ações específicas de reorganização do fluxo e melhoria da qualidade da assistência perinatal vêm sendo desencadeadas pela Comissão Perinatal desde 1999. Rotinas de assistência ao pré-natal, acolhimento imediato da gestante e sua vinculação à maternidade desde o início do pré-natal, acesso ao pré-natal de alto risco pela Central de Marcação de Consultas, ampliação de leitos de cuidados intensivos neonatais e estruturação do ambulatório de acompanhamento do recém-nascido de risco foram estabelecidos com conseqüente redução na mortalidade neonatal precoce. Ou seja, recém-nascidos com graves afecções perinatais estão sobrevivendo por mais tempo e aumentando as taxas da mortalidade pós-neonatal. É possível que posteriores melhoras na qualidade do pré-natal e no atendimento ao parto também reduzam essas afecções no período pósneonatal, pois são causas potencialmente evitáveis em sua maioria. Já em 1994, os Estados Unidos apresentavam taxa de mortalidade por afecções perinatais no período pós-neonatal de 12,5 (em brancos) e 53,9 (em negros) por $100.000 \mathrm{NV}, 21$ muito inferiores às de Belo Horizonte em 2002-2004.

O importante decréscimo das taxas de mortalidade por meningites/encefalites pode ser atribuído ao provável impacto da implantação da vacina antihaemophilus e da vacina triviral pelo Programa Nacional de Imunizações do Ministério da Saúde.

É preocupante o aumento de óbitos por causas mal definidas, com taxas também crescentes. Entre as mal definidas, predominou o código R99, que é inespecífico, e pode incluir também óbitos sem assistência médica. Verificou-se que cerca de 30\% dos óbitos por causas mal definidas de Belo 
Horizonte foram domiciliares (dados não apresentados), o que chama a atenção, por terem ocorrido em município com ampla cobertura hospitalar. Sabese que a proporção de óbitos por causas mal-definidas é um indicador da qualidade das informações sobre causas de óbito 22 e sua ocorrência aponta para a necessidade de melhoria da assistência, de investimentos em recursos diagnósticos e terapêuticos e de treinamento dos profissionais para o preenchimento correto da Declaração de Óbito (DO). Com a implantação do Comitê de Investigação do Óbito Infantil e Perinatal em Belo Horizonte, em 2002, esperava-se maior impacto sobre essas causas, já que constitui uma atribuição dos comitês a melhor qualificação das informações. ${ }^{4}$ É provável, entretanto, que a não incorporação dos resultados das investigações feitas pelo comitê às estatísticas oficiais do SIM do município tenha contribuído para essa ocorrência (Ishitani LH, informação pessoal). Por outro lado, deve ser ressaltado que o aumento dessas causas reforça a necessidade de implantação de um Serviço de Verificação de Óbitos (SVO) em Belo Horizonte para esclarecimento desses óbitos e redirecionamento de ações preventivas.

As taxas de mortalidade por causas externas tiveram pequeno acréscimo no período. Chamou a atenção o fato de a maioria dessas mortes terem sido devidas à aspiração (W78, W79 e W84 do capítulo de causas externas), que são causas potencialmente evitáveis de óbito, 23 pois sabe-se que o reforço das orientações para posicionamento das crianças após alimentação pode ser decisivo para a redução do

\section{Referências}

1. Jones G, Steketee RW, Black RE, Bhutta ZA, Morris SS, Bellagio Child Survival Study Group. How many children can we prevent this year? Lancet. 2003; 362: 65-71.

2. Victora CG. Intervenções para reduzir a mortalidade infantil, pré-escolar e materna no Brasil. Rev Bras Epidemiol. 2001; 4: 3-61.

3. Brasil. Ministério da Saúde. Secretaria de Vigilância em Saúde. Mortalidade infantil no Brasil: determinantes e desigualdade. In: Brasil. Ministério da Saúde. Secretaria de Vigilância em Saúde. Saúde Brasil 2006: uma análise da desigualdade em saúde. Brasília, DF; 2006. p. 251-304.

4. Brasil. Ministério da Saúde. Secretaria de Atenção à Saúde, Secretaria de Vigilância em Saúde. Manual dos Comitês de Prevenção do Óbito Infantil e Fetal. Brasília, DF; 2004.

5. Brasil. Ministério da Saúde. Secretaria de Vigilância em Saúde. Saúde. Evolução da mortalidade infantil. In: Brasil. Ministério da Saúde. Secretaria de Vigilância em Saúde. Brasil 2004: uma análise da situação de saúde. Brasília, DF; 2004. p. 120-33. expressivo percentual de mortes secundárias à inalação de conteúdo gástrico.

\section{Conclusões}

Em síntese, os achados desse estudo indicam ocorrência de mudança significativa no padrão de causas de mortes infantis pós-neonatais. É provável que o declínio mais lento das taxas de mortalidade pósneonatal nos últimos anos tenha ocorrido devido ao aumento da contribuição das malformações congênitas (que passaram a representar a principal causa de óbitos pós-neonatais no último triênio), e das afecções perinatais. A predominância dessas causas de óbito mais dificilmente evitáveis no período pósneonatal ao invés do tradicional grupo doenças infecciosas doenças respiratórias aponta para um perfil de causas semelhante ao de países desenvolvidos.

Entretanto, apesar da expressiva queda das taxas e da mudança no perfil de causas, a ocorrência de cerca de um quarto das mortes infantis pós-neonatais por diarréia, pneumonia, desnutrição e septicemias é motivo de preocupação. Tanto essas, quanto as mortes por aspiração e as mal definidas precisam ser melhor investigadas, pois certamente muitos destes óbitos são potencialmente evitáveis com ações de promoção de saúde, diagnóstico e tratamento oportuno. Somente com a intensificação dos esforços para a melhor compreensão desses eventos, intervenções adequadas poderão ser direcionadas para a minimização do risco de ocorrência dessas mortes precoces.
6. Caldeira AP, França E, Perpetuo IHO, Goulart EMA. Evolução da mortalidade infantil por causas evitáveis, Belo Horizonte, 1984-1998. Rev Saúde Pública. 2005; 39: 6774.

7. Malta DC, Merhy EE. A avaliação do Projeto Vida e do acolhimento no Sistema Único de Saúde de Belo Horizonte. Rev Min Enferm. 2004; 8: 259-67.

8. Lansky S, Passos C, Mendonça ML, Silva ME, Reis R, Oliveira RC, Rodrigues C, Rates S, Mayer R. Comitê de Prevenção do Óbito Infantil e Perinatal da Secretaria Municipal de Saúde de Belo Horizonte. Saúde digital, agosto 2003. Disponível em: htpp//www.pbh.gov.br/smsa/ biblioteca/saudedigital/agosto2003/sonialansky3.html. [2007 maio 31].

9. Côrtes MCJW, Rezende EM, Martins EF, França E, Lansky, S. Monitoramento do óbito infantil e fetal em Belo Horizonte: experiência da parceria entre a Universidade Federal de Minas Gerais e a Secretaria Municipal de Saúde. Rev Med Minas Gerais. 2006; 16 (Supl 2): S52-S5. 
10. Lansky S. A gestão da qualidade e da integralidade do cuidado à saúde para a mulher e a criança no SUS-BH: a experiência da Comissão Perinatal. In: I Expogest-Mostra Nacional de Experiências Inovadoras na Gestão do SUS; 2006 4-7 jun.; Brasília, DF. Brasília, DF: Ministério da Saúde; 2006. Disponível em: htpp://dtr2002.saude.gov.br/ expogest/ Trabalhos premiados.htm. [2007 maio 31].

11. Ortiz LP. Utilização das causas evitáveis na mortalidade infantil como instrumento de avaliação das ações de saúde. In: Anais do $\mathrm{X}$ Encontro Nacional de Estudos Populacionais; 1996 7-11 out.; Caxambu, MG. Belo Horizonte: ABEP; 1996. v. 4, p. 2253-68.

12. França E. Estudo de determinantes da mortalidade infantil por diarréia, pneumonia e desnutrição na Região Metropolitana de Belo Horizonte em 1991-1992 [tese doutorado]. Belo Horizonte: Faculdade de Medicina da Universidade Federal de Minas Gerais; 1995.

13. Rutstein DD, Berenberg W, Chalmers TC, Child CG, Fishman AP, Perrin EB. Measuring the quality of medical care: a clinical method. N Engl J Med. 1976; 294: 582-8.

14. OMS (Organização Mundial de Saúde). Centro Colaborador da OMS para Classificação das Doenças em Português. Classificação Estatística Internacional de Doenças e Problemas Relacionados à Saúde. 10 revisão. São Paulo: EDUSP; 1995.

15. Brasil. Ministério da Saúde. Mortalidade CID 10-lista de tabulação CID_BR. Disponível em: http// tabnet.datasus. gov.br/cgi/sim/obtcid10br.htm. [2007 jun 6].
16. França E, Souza JM, Guimarães MDC, Goulart EMA, Colosimo E, Antunes CMF. Associação entre fatores socioeconômicos e mortalidade infantil por diarréia, pneumonia e desnutrição em região metropolitana do sudeste do Brasil: um estudo caso-controle. Cad Saúde Pública. 2001; 17: 1437-47.

17. Rezende EM. Análise multidimensional de causas múltiplas de óbitos humanos em Belo Horizonte, 1998 [tese doutorado]. Belo Horizonte: Faculdade de Medicina da Universidade Federal de Minas Gerais; 2003.

18. Kochanek KD, Martin JA. Supplemental analyses of recent trends in infant mortality. Int J Health Serv. 2005; 35: 10115 .

19. Belo Horizonte. Secretaria de Saúde. Projeto de Reorganização da Assistência Pública às Doenças Respiratórias. Belo Horizonte; 1986.

20. Belo Horizonte. Secretaria de Saúde. Programas de Prevenção e Combate à Desnutrição. Belo Horizonte; 1993

21. Scott CL, Iyasu S, Rowley D, Atrash HK. Postneonatal mortality surveillance - United States, 1980-1994. MMWR CDC Surveill Summ. 1998; 47: 15-28.

22. OPAS (Organización Panamericana de la Salud). Sobre la estimación de taxas de mortalidad para países de la Región de las Américas. Bol Epidemiol. 2003; 24: 1-5.

23. Tomashek KM, Hsia J, Iyasu S. Trends in post-neonatal mortality attributable to injury, United States, 1988-1998. Pediatrics. 2003; 111: 1219-25.

Recebido em 17 de novembro de 2006

Versão final apresentada em 12 de junho de 2007

Aprovado em 7 de agosto de 2007 